Rev. Biol. Trop., 46(3):791-803, 1998

\title{
Variables asociadas con el uso de hábitat del manatí del Caribe (Trichechus manatus), en Quintana Roo, México (Mammalia)
}

Javier Axis-Arroyo ${ }^{1}$, Benjamín Morales-Vela², Daniel Torruco-Gómez y María Eugenia VegaCendejas ${ }^{1}$

1 Centro de Investigaciones y de Estudios Avanzados del IPN, Unidad Mérida; Carretera antigua a Progreso Km 6, C. P. 97310 A.P. 73 Cordemex, Mérida; Yucatán México. Internet: axis@kin.cieamer.conacyt.mx

2 El Colegio de la Frontera Sur, Unidad Chetumal. Zona Industrial \# 2 Carretera Chetumal-Bacalar, C.P: 77000 A.P. 424 Chetumal, Quintana Roo; México.

Recibido 26-IX-1996. Corregido 20-IV-1998. Aceptado 29-IV-1998.

\begin{abstract}
Influence of atmospheric variables and water temperature, winds, cloudiness, depth, salinity, grass and algal abundance, and group structure on the spatial distribution of Trichechus manatus manatus was studied in Chetumal Bay, Quintana Roo, Mexico. Boat surveys were done from November 1994 through June 1995. There was little association with cloudiness and atmospheric and water temperature (in contrast with reports for the Florida subspecies, Trichechus manatus latirostris); the variables with moderate association were salinity (as in Florida), depth (frequent use of depths between $0.80-2 \mathrm{~m}$ ) and group structure (which suggests that the zone is not an important mating area). Spatial distribution was more associated with drastic changes in wind intensity and the available food (similar to previous works in Mexico).
\end{abstract}

Key words: Manatee, habitat, Mexico, path analysis, spatial distribution.

El manatí del Caribe es una especie conformada por dos subespecies: el manatí de Flori$\mathrm{da}$ (Trichechus manatus latirostris), que se distribuye en la Península de Florida, Estados Unidos y el manatí de las Antillas (Trichechus manatus manatus), que se distribuye desde el Estado de Tabasco en México, hasta Recife en Brasil (Reynolds 1979, Bonde 1984, Domming y Hayek 1986, Reynolds y Odell 1991, Jefferson et al. 1993, Marsh y Lefebre 1994). Aunque su distribución ha sido ampliamente estudiada los estudios sobre la ecología de la especie se ha limitado a la subespecie de Florida y se a generalizado sin considerar que $T$. $m$. latirostris se distribuye en zonas subtropicales mientras que T. m. manatus en zonas tropicales, por lo que el presente trabajo busca conocer las variables que afectan el uso de hábitat de la especie en una zona del Caribe (la Bahía de Chetumal, Quintana Roo, México), teniendo como base comparativa los trabajos realizados con la subespecie de Florida, lo cual nos permitirá tener una referencia sobre que variables afectan los movimientos de la especie en zonas tropicales y sobre las ventajas o riesgos de la generalización de los conocimientos obtenidos con las poblaciones de zonas subtropicales. 
Los estudios realizados con $T$. m. latirostris reportan como limitantes de su distribución en un área a los siguientes factores: temperatura del agua (Husar 1977, Whitehead 1977, Campbell e Irvine 1981, Shane 1981, Irvine 1983, Reynolds y Wilcox 1986), Salinidad (Lluch 1965, Husar 1977, Rose 1985), cantidad y tipo de alimento disponible (Hartman 1979, Bengtson 1983, Etheridge et al. 1985), profundidad de los cuerpos de agua (Hartman 1979, Powell y Rathbun 1984) y actividad humana (O' Shea et al. 1984, 1985, Rose 1985).

Considerando las diferencias climáticas entre las zonas de distribución de la especie (tropical y subtropical) y los fenómenos meteorológicos más comunes en el Caribe Mexicano (nortes, huracanes y ciclones), se decidió incluir las variables: temperatura atmosférica, vientos y nubosidad; como posibles limitantes de la distribución.

El manatí es una especie de hábitos solitarios, no agresivos ni territorialistas y aunque llegan a agruparse en forma casual, la formación de grupos es fundamentalmente con fines reproductivos (Reynolds 1981), por lo que el agrupamiento en un área puede ser atribuido a características etológicas de la especie y no a los atributos ecológicos de la zona, en consecuencia, la formación de grupos (agrupamiento) se incluyó como otra variable que influye en el uso de hábitat de la especie. Por lo que las variables que se consideran en el presente estudio son: temperatura atmosférica, vientos, nubosidad, profundidad, salinidad, temperatura del agua, abundancia de pastos y algas y agrupamiento.

Los estudios realizados por Zárate (1993) y Morales y Olivera (1992, 1994), compararan los censos realizados en el Caribe Mexicano y en el área de mayor presencia de manatíes en el Mar Caribe, la Costa de Belice, observando una alta similitud entre los índices de abundancia relativa de la Bahía de Chetumal y de Belice, por lo que consideran a la Bahía de Chetumal como parte del hábitat más importante para el manatí en el Caribe.

\section{MATERIALES Y METODOS}

El estudio se realizó en la Bahía de Chetumal, localizada en el extremo sureste del Estado de Quintana Roo, México, Tiene un área de 1 $090 \mathrm{~km}^{2}$ (Chavira et al. 1992), su costa noroeste tiene una extensión de $44 \mathrm{~km}$ desde la Ciudad. de Chetumal hasta Río Creek (Anónimo 1980). Se caracteriza por tener: una temperatura del agua mayor a $20^{\circ} \mathrm{C}$ en el mes más frío del año, salinidad variable por numerosas efluentes naturales de agua dulce, profundidad de $4 \mathrm{~m}$ en promedio, grandes extensiones someras, amplias zonas con pastos marinos, algas y otros tipos de vegetación acuática y una alta diversidad de hábitats (lagunas, canales de marismas y cayos arenosos cubiertos de mangle).

Con la finalidad de hacer comparativo el presente estudio con otros trabajos realizados en la Bahía de Chetumal, se ha respetado la división propuesta por Morales y Olivera (1992), que divide la bahía en dos grandes zonas: la línea de costa (de la zona de mareas hasta $2 \mathrm{~km}$ de la costa) y el centro de la bahía, de estas, la primera es la de mayor importancia para los manatíes, ya que los avistamientos reportados en el centro de la bahía son escasos y generalmente corresponden a individuos en tránsito. Del área costera, la parte noreste ha sido considerada como la de mayor presencia de individuos en la zona (Zárate 1993) y la de mayor importancia para las hembras con crías (Morales y Olivera 1994); por lo que para el presente trabajo se aplicó un esfuerzo de búsqueda constante en la costa noreste y se monitoreó el resto de la bahía.

El trabajo de campo se efectuó de noviembre de 1994 a junio de 1995, en dos de las tres temporadas climáticas del año en la zona: la temporada de nortes (TN) noviembre de 1994 -febrero de 1995; y la temporada de secas (TS) marzo - junio de 1995, no analizándose la temporada de lluvias por probiemas de financiamiento.

Considerando los estudios realizados en la zona sobre distribución de la especie (Colmenero et al. 1988, Fuentes y Aguayo 1989, Morales y Olivera 1992, Zárate 1993, Morales y Olivera 1994) se seleccionaron ocho áreas en TN y tomando los resultados de los primeros 
muestreos, nueve áreas en TS. Las áreas fueron delimitadas arbitrariamente considerando características topográficas y ecológicas como cambios drásticos en la salinidad del agua o en el patrón de corrientes (Fig. 1a). Para confirmar la importancia de las áreas muestreadas se monitoreó el resto de la bahía efectuando el siguiente esfuerzo de búsqueda durante el desarrollo de la investigación: 6 recorridos en lancha de toda la franja costera, dos censos aéreos de toda la bahía y uno de la costa noroeste.

El muestreo se realizó en 14 campañas de campo de cinco días consecutivos de duración con frecuencia quincenal (las áreas de la uno a la siete se muestrearon en 14 salidas en ambas temporadas; la ocho en siete salidas en TN y la nueve y 10 en siete salidas en TS), el esfuerzo de búsqueda de manatíes en cada área por día, varió según el número de avistamientos registrados debido al tiempo necesario para la identificación de clases de edades de los ejemplares avistados (de 1.5 a 4 h).

Mediante recorridos en lancha se registraron: observaciones de manatíes (mediante barrido de área de transectos lineales según la técnica etológica de Altmann 1974), salinidad (con refractómetro de mano con precisión de uno), temperatura del agua (con termómetro de campo con escala de $0^{\circ} \mathrm{C}$ a $50^{\circ} \mathrm{C}$ y una precisión de $0.5^{\circ} \mathrm{C}$ ), y profundidad (por plomada a fondo, con precisión de $1 \mathrm{~cm}$ ). Las mediciones se tomaron en 48 puntos fijos (Fig. 1b) y en cada lugar en donde se observaba la presencia de manatíes. Los datos meteorológicos se obtuvieron por día del reporte meteorológico de la Estación 750, Chetumal, Q. Roo. de la Dirección General del Sistema Meteorológico Nacional de México.

Para reducir los sesgos generados por la diferencia en el tiempo dedicado a cada área, los avistamientos fueron analizados utilizando un índice de abundancia relativa (IAR), que es igual al número de manatíes avistados en un área, entre el tiempo del esfuerzo de búsqueda ejercido en el área. El agrupamiento se evaluó mediante la probabilidad de que un manatí avistado este acompañado de al menos otro individuo (probabilidad de agrupamiento), cuando todos los individuos avistados en un área conformaban un grupo la probabilidad fue uno, cuanto los manatíes avistados estaban solitarios la probabilidad fue cero (como la relación madre-cría es la única relación permanente de la especie, para el análisis de agrupamiento cada pareja de madre-cría se consideró como una unidad).

Debido a los pocos (o nulos) avistamientos efectuados en algunas áreas, los muestreos de vegetación se efectuaron en las siete áreas de mayor presencia de manatíes en ambas temporadas (áreas uno a siete). Las recolectas se realizaron en puntos aledaños a los utilizados en el muestreo de salinidad y temperatura utilizando el método de cosecha total de área en un cuadrante de $0.5 \mathrm{~m}^{2}$ por buceo libre en zonas con profundidad $<4 \mathrm{~m}$ y con buceo SCUBA en profundidades $>4 \mathrm{~m}$, se colectó un cuadrante con dos repeticiones y se reportaron valores promedios. Las especies se identificaron siguiendo las claves de Wynne 1985, Lot 1991 y Norris y Bucher 1992; la biomasa se cuantificó en peso seco (secado de las muestras a $90^{\circ} \mathrm{C}$ durante $6 \mathrm{~h}$ ) y a partir de ella se estimó la dominancia de las especies utilizando el índice de dominancia simple (IDS) y el índice biológico de Sanders (IBS) (Pielou 1977).

La modelación de los resultados se efectuó mediante el análisis de dirección (Path analysis), el diagrama causal (Fig. 2) entre variables consideradas y la presencia de un manatí en un área se validó por el procedimiento estadístico de aproximación a $\mathrm{c}^{2}$ propuesto por Mitchell (1992), a un nivel de significación de 0.05 .

\section{RESULTADOS}

Avistamientos y agrupamiento de manatíes: Se avistaron 177 manatíes en ambas temporadas en toda la bahía, de estos el $85.31 \%$ (151 manatíes) se avistaron en las 10 áreas seleccionadas y el 14.69\% (26 manatíes) en el resto de la bahía. El número de avistamientos y el IAR por temporada (Cuadro 1), se analizó considerando cada una de las áreas por cada salida 


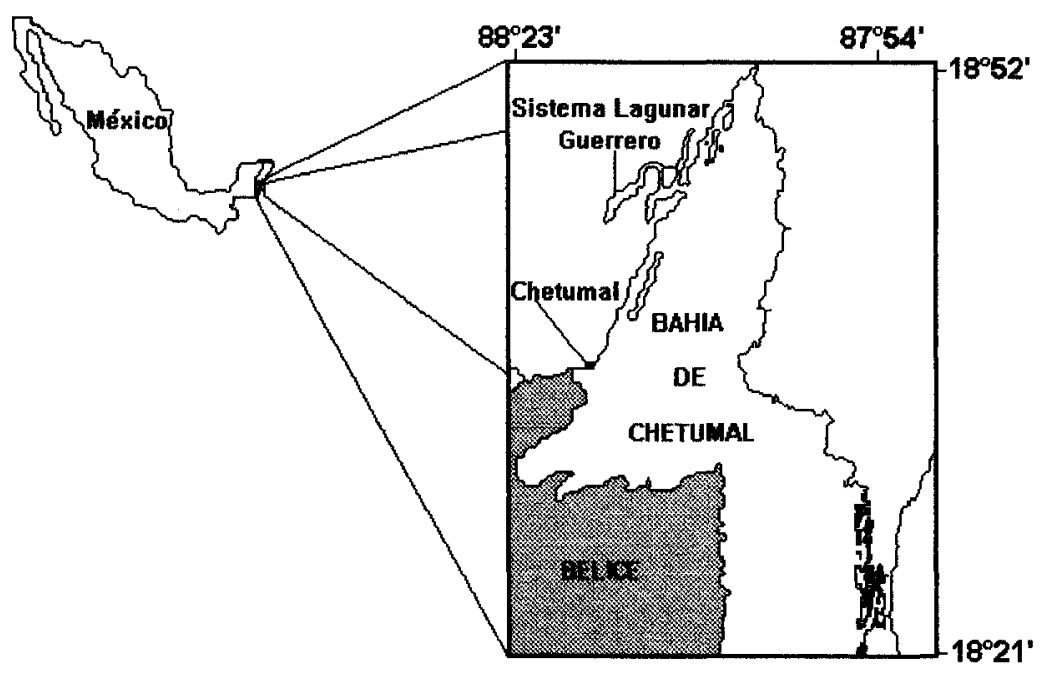

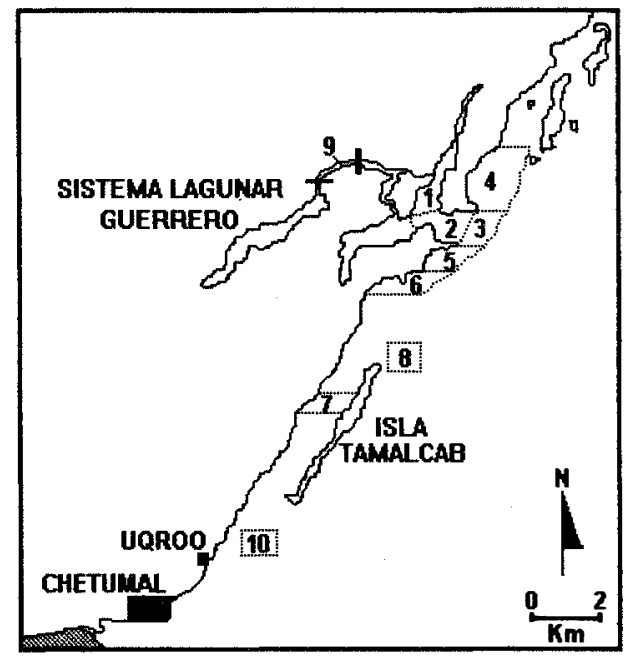

1A

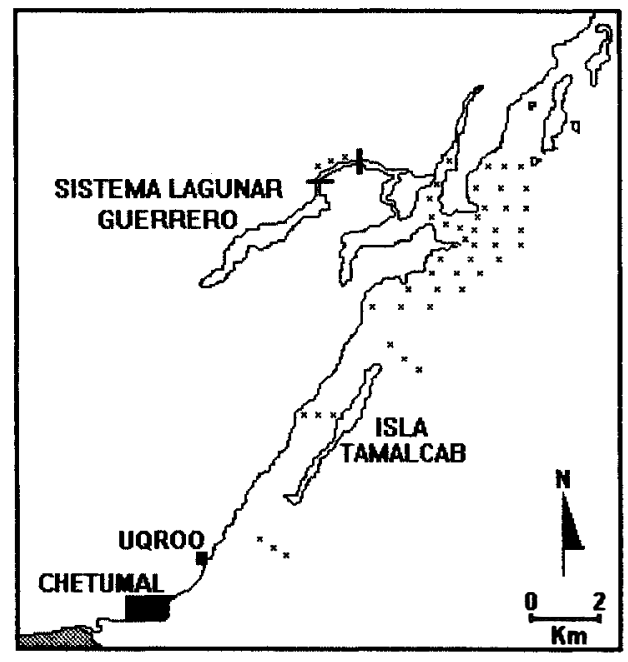

1B

Fig. 1. Ubicación de las estaciones muestrales. 1a.- áreas muestrales. 1b.- puntos fijos de muestreo de las variables: profundidad, salinidad, temperatura del agua y abundancia de pastos y algas.

en forma individual, lo que originó una abundancia de valores cero que sesgo la distribución muestral, por lo que se decidió aplicar la prueba de Kruskall-Wallis (ANDEVA no paramétrica) para comparar áreas; no encontrándose diferencias significativas entre áreas en ambas temporadas (Kruskall-Wallis $p=0.044$ en TN y $p=0.291$ en TS), las áreas con IAR mas altos fueron la uno y dos en TN y uno y 10 en TS. La probabilidad de agrupamiento por temporada (Cuadro 2), no reportó diferencias significativas entre temporadas (Wilcoxon $\mathrm{p}=0.753$ ) $\mathrm{ni}$ entre áreas (Wilcoxon $p=0.612$ ), las áreas de mayor probabilidad de agrupamiento fueron la cuatro, la uno y la siete. 


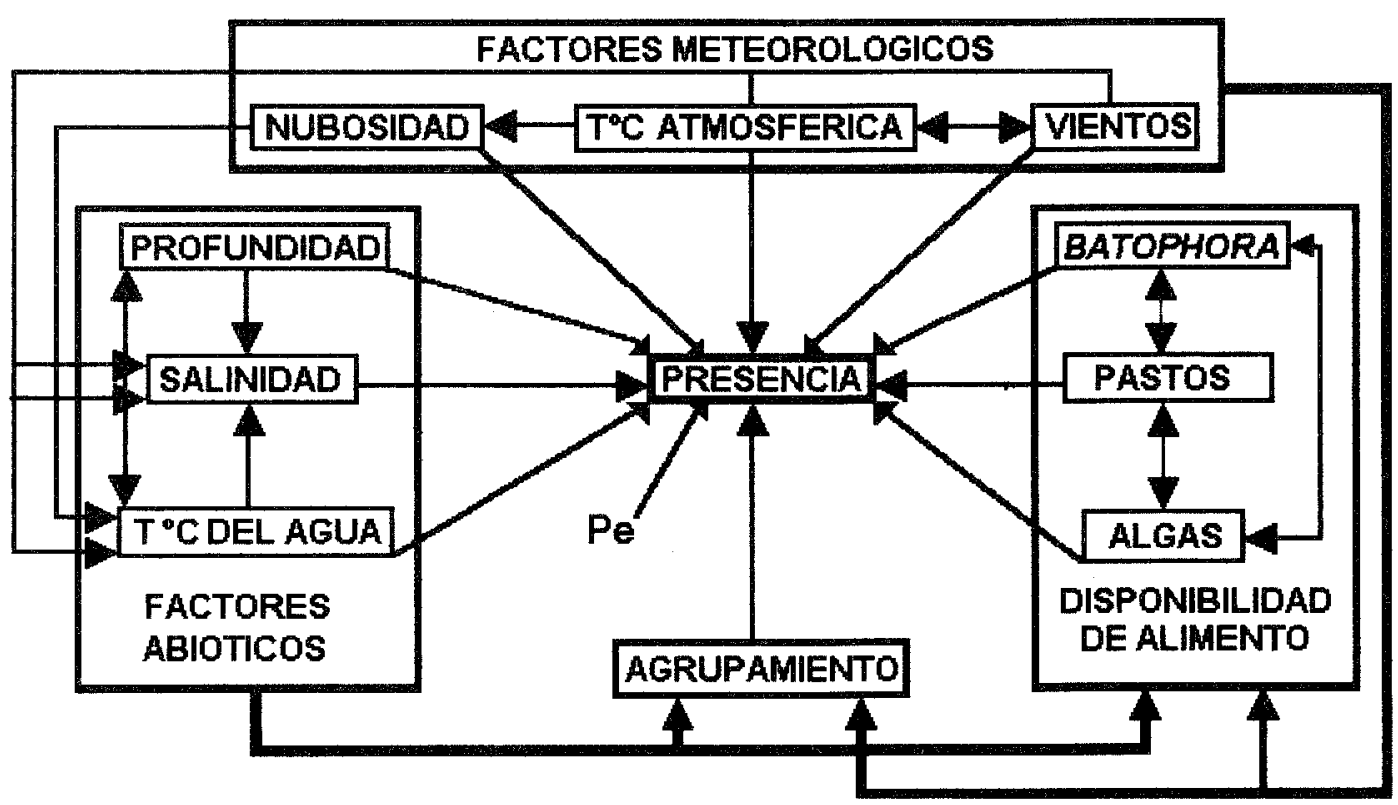

Fig. 2. Diagrama causal de relaciones entre variables analizadas. Las flechas delgadas representan la relación directa de una variable sobre otra y las flechas gruesas la influencia de todo un bloque de variables sobre otro.

Parámetros físico-químicos: Las concentraciones de salinidad (Cuadro 3), registraron variaciones significativas entre áreas (ANDEVA $p<0.000$ ), y entre meses (ANDEVA $p<$ 0.000 ). La variación máxima corresponde al área nueve (ANDEVA $p<0.000$ ) y a mayo (ANDEVA $p=0.078$ ), la variación mínima se observó en el área seis (ANDEVA $p=0.853$ ), y en enero (ANDEVA $p=0.623$ ). Las áreas con valores de mayor similitud correspondieron a la zona de la bahía próxima a la boca de la barra (áreas tres, cuatro y cinco) y las de menor similitud a aquellas en la que los valores de salinidad son extremos (área nueve, de menor salinidad y área 10, de mayor salinidad). En relación con la temperatura del agua (Cuadro 3) no se encontraron variaciones significativas entre áreas (ANDEVA $p=0.666$ ), pero sí entre meses (ANDEVA $p<0.000$ ). Las variaciones mínimas se observan en el área $10($ ANDEVA $p=0.985)$ y en diciembre (ANDEVA $p=0.180$ ), las máximas corresponden al área ocho (ANDEVA $p=0.904$ ) y a enero (ANDEVA $p<0.000$ ), en todo el período de estudio no se registraron temperaturas menores de $20^{\circ} \mathrm{C}$.

Vegetación: La biomasa de la vegetación recolectada en cada área (Cuadro 4), fue comparada por temporada considerando las medias y las desviaciones estándar de los pesos de cada especie en cada punto muestral, no encontrándose diferencias estadísticas significativas entre temporadas ( $t$ muestras pareadas $p<0.001)$. Analizando la dominancia de cada especie (Cuadro 5), Batophora sp. presenta una amplia dominancia sobre el resto de la vegetación (IDS $=92.17 \%, \mathrm{IBS}=34.62 \%$ ), por lo que se decidió considerarla dentro del análisis de dirección como una variable independiente del grupo de algas.

Variables meteorológicas: Para el análisis de los factores meteorológicos se consideró las variaciones diarias en los días de muestreo, considerando los valores mínimos máximos y la media diaria, estimandose en porcentaje con relación enl promedio de los tres días anteriores (Cuadro 6), observando màyores variaciones en 


\section{CUADRO 1}

Total de avistamientos, esfuerzo de búsqueda e índice de abundancia relativa

\section{Temporada}

\begin{tabular}{|c|c|c|c|c|c|c|}
\hline \multirow[b]{2}{*}{ Area } & \multicolumn{3}{|c|}{ Nortes } & \multicolumn{3}{|c|}{ Secas } \\
\hline & Esfuerzo* & Avistamientos & IAR & Esfuerzo & Avistamientos & IAR \\
\hline 1 & 12: 00 & 24 & 2.00 & 7:00 & 16 & 2.28 \\
\hline 2 & $32: 45$ & 34 & 1.03 & 28: 15 & 18 & 0.63 \\
\hline 3 & $22: 45$ & 9 & 0.39 & $21: 30$ & 2 & 0.09 \\
\hline 4 & $17: 45$ & 2 & 0.11 & 10: 00 & 6 & 0.60 \\
\hline 5 & 8: 30 & 0 & 0.00 & 5: 00 & 0 & 0.00 \\
\hline 6 & $8: 30$ & 1 & 0.11 & $5: 30$ & 1 & 0.18 \\
\hline 7 & $16: 10$ & 11 & 0.67 & 18: 45 & 2 & 0.11 \\
\hline 8 & $16: 25$ & 1 & 0.06 & - & - & - \\
\hline 9 & - & - & - & $20: 15$ & 4 & 0.20 \\
\hline 10 & - & - & - & $7: 00$ & 14 & 2.00 \\
\hline
\end{tabular}

IAR (índice de abundancia relativa).-Número de avistamientos por hora de esfuerzo de búsqueda. *.- En horas de búsqueda.

\section{CUADRO 2}

Conformación de grupos de manatíes avistados y probabilidad de agrupamiento*

Temporada

\begin{tabular}{|c|c|c|c|c|}
\hline \multirow[b]{2}{*}{ Area } & \multicolumn{2}{|l|}{ Nortes } & \multicolumn{2}{|l|}{ Secas } \\
\hline & $\begin{array}{l}\text { Media de individuos } \\
\text { por grupo avistado }\end{array}$ & $\begin{array}{l}\text { Probabilidad de } \\
\text { agrupamiento }\end{array}$ & $\begin{array}{l}\text { Media de individuos por } \\
\text { grupo avistado }\end{array}$ & $\begin{array}{l}\text { Probabilidad } \\
\text { agrupamient }\end{array}$ \\
\hline 1 & $6.0 \pm 3.36$ & 1.00 & $3.5 \pm 1.91$ & 0.97 \\
\hline 2 & $5.8 \pm 3.56$ & 0.91 & $3.0 \pm 1.00$ & 0.76 \\
\hline 3 & $3.0 \pm 1.41$ & 0.77 & 0 & 0.63 \\
\hline 4 & $2.0 \pm 0.01$ & 1.00 & $3.0 \pm 1.41$ & 1.00 \\
\hline 5 & 0 & - & 0 & - \\
\hline 6 & 0 & - & 0 & - \\
\hline 7 & $3.5 \pm 3.5$ & 0.81 & $2.0 \pm 0.00$ & 0.84 \\
\hline 8 & 0 & - & - & - \\
\hline 9 & - & - & $2.0 \pm 0.00$ & 0.40 \\
\hline 10 & - & - & $7.0 \pm 0.00$ & 1.00 \\
\hline
\end{tabular}

*.- Probabilidad de que un manatí avistado este acompañado, = 1 cuando todos los individuos en un área conformaban un grupo, $=0$ cuanto fueron solitarios. 


\section{CUADRO 3}

Valores promedio de salinidad y temperatura del agua

Temporada

$\begin{array}{ccccccc}\text { Area } & \text { Salinidad } & \begin{array}{c}\text { Nortes } \\ \text { Temperatura* }\end{array} & \text { N } & \text { Salinidad } & \begin{array}{l}\text { Secas } \\ \text { Temperatura }\end{array} & \text { N } \\ 1 & 12.6 \pm 2.43 & 27.3 \pm 0.49 & 18 & 15.0 \pm 1.58 & 15.0 \pm 1.58 & 39 \\ 2 & 11.0 \pm 0.74 & 26.9 \pm 0.31 & 22 & 13.8 \pm 1.24 & 13.8 \pm 1.24 & 67 \\ 3 & 11.8 \pm 0.60 & 27.0 \pm 1.14 & 28 & 13.4 \pm 1.05 & 13.4 \pm 1.05 & 102 \\ & & & & & & \\ 4 & 11.5 \pm 0.28 & 28.5 \pm 0.33 & 24 & 13.3 \pm 1.22 & 13.3 \pm 1.22 & 77 \\ 5 & 11.8 \pm 0.81 & 26.6 \pm 2.27 & 24 & 13.1 \pm 1.18 & 13.1 \pm 1.18 & 77 \\ 6 & 11.7 \pm 0.24 & 28.2 \pm 0.40 & 16 & 13.2 \pm 1.20 & 13.2 \pm 1.20 & 77 \\ 7 & 11.1 \pm 1.72 & 26.7 \pm 2.34 & 16 & 13.4 \pm 1.06 & 13.4 \pm 1.06 & 54 \\ & & & & & & \\ 8 & 10.4 \pm 3.07 & 26.8 \pm 2.67 & 6 & - & - & - \\ 9 & 3.5 \pm 1.41 & 28.3 \pm 0.62 & 6 & 8.4 \pm 1.00 & 8.4 \pm 1.00 & 52 \\ 10 & - & - & - & 14.2 \pm 0.64 & 14.2 \pm 0.64 & 39\end{array}$

*.- En grados centígrados.

N.- Mediciones realizadas

\section{CUADRO 4}

Peso de la vegetación

\section{Temporada}

\begin{tabular}{|c|c|c|c|c|c|c|c|}
\hline \multirow[b]{2}{*}{ Area } & \multirow[b]{2}{*}{ Especie } & \multicolumn{3}{|c|}{ Nortes } & \multicolumn{3}{|c|}{ Secas } \\
\hline & & Media & DS* & $\mathrm{N}$ & Media & DS* & $\mathrm{N}$ \\
\hline \multirow[t]{5}{*}{1} & Batophora sp. & 3.66 & 8.24 & 12 & 4.18 & 4.00 & 12 \\
\hline & Halodule wrightii & 1.17 & 2.14 & 12 & 1.84 & 1.84 & 12 \\
\hline & Chara sp. & 0.41 & 0.98 & 12 & 1.25 & 0.90 & 12 \\
\hline & Naja sp. & 0.94 & 0.98 & 12 & 2.43 & 1.59 & 12 \\
\hline & Total del área & 6.19 & 1.00 & 12 & 9.82 & 8.35 & 12 \\
\hline \multirow[t]{6}{*}{2} & Batophora sp. & 15.55 & 18.75 & 21 & 15.78 & 14.22 & 21 \\
\hline & Halodule wrightii & 1.71 & 2.48 & 21 & 1.20 & 3.84 & 21 \\
\hline & Thalassia testudinum & 0.38 & 0.49 & 21 & 2.15 & 3.48 & 21 \\
\hline & Chara sp. & 1.22 & 2.32 & 21 & 1.25 & 2.29 & 21 \\
\hline & Naja sp. & 0.03 & 0.07 & 21 & 0.05 & 0.09 & 21 \\
\hline & Total del área & 18.90 & 18.24 & 21 & 20.45 & 15.90 & 21 \\
\hline \multirow[t]{4}{*}{3} & Batophora sp. & 160.30 & 125.82 & 33 & 269.31 & 113.11 & 33 \\
\hline & Halodule wrightii & 2.87 & 4.89 & 33 & 2.86 & 6.48 & 33 \\
\hline & Thalassia testudinum & 1.37 & 3.02 & 33 & 1.38 & 2.05 & 33 \\
\hline & Total del área & 164.55 & 127.57 & 33 & 273.55 & 105.39 & 33 \\
\hline
\end{tabular}




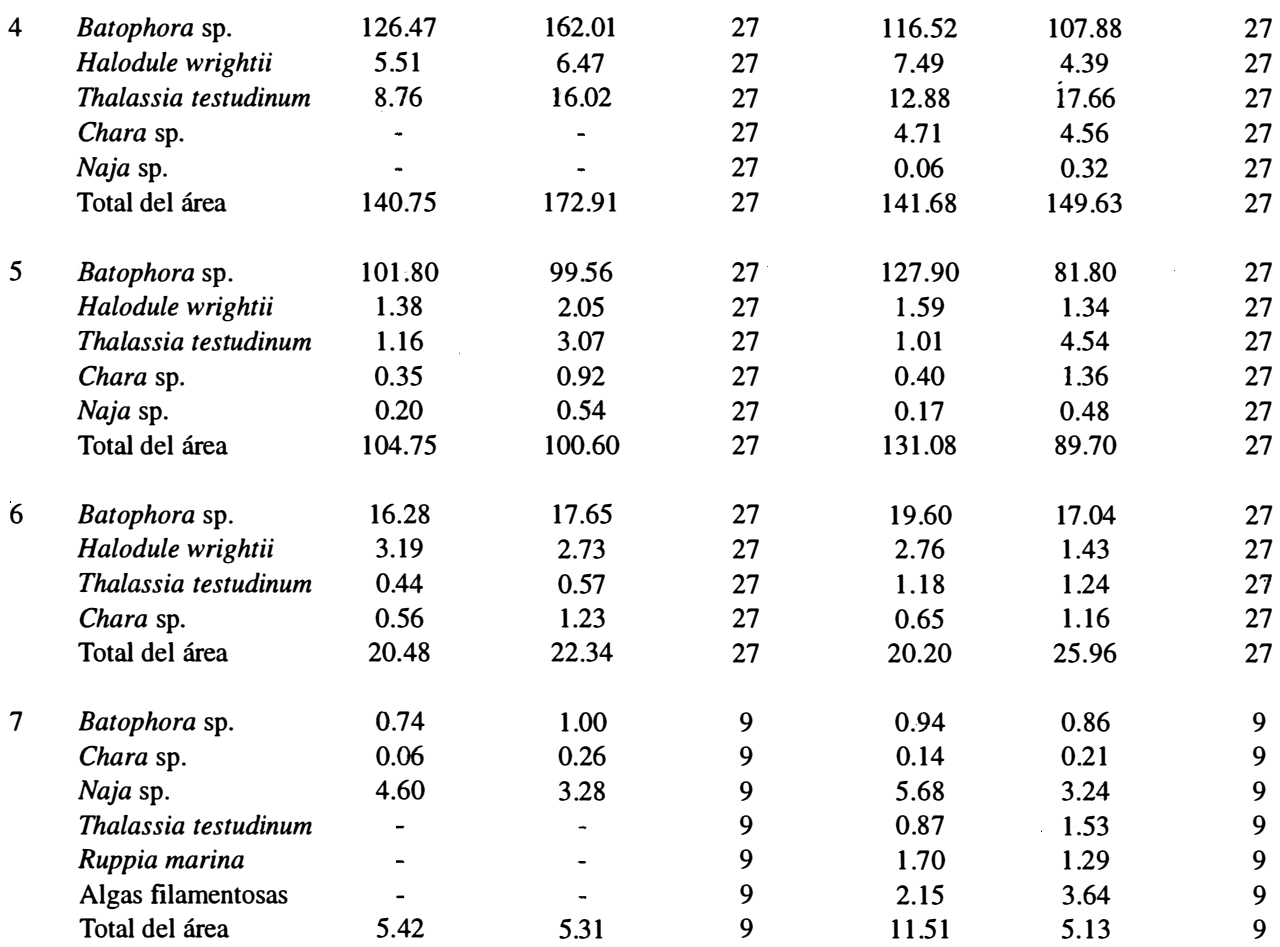

Peso en g. de peso seco/ $\mathrm{m}^{2}$

*.- Desviación estándar

N.- Mediciones realizadas

\section{CUADRO 5}

Dominancias biológicas de la vegetación

Temporada

Nortes

Secas

$\quad$ Especies
Batophora sp.
Thalassia testudinum
Halodule wrightii
Chara sp.
Naja sp.
Algas filamentosas
Ruppia marina

$\begin{array}{cc}\text { IBS } & \text { CSB } \\ 36.23 & 95.10 \\ 12.84 & 2.50 \\ 24.42 & 3.40 \\ 12.87 & 0.70 \\ 13.85 & 1.30 \\ - & - \\ - & -\end{array}$

IBS

CSB

IBS.- Indice biológico de Sanders

CSB.- Coeficiente simple de dominancia. 


\section{CUADRO 6}

Medias de los factores meteorológicos en los días en que se realizaron los muestreos y porcentaje de variación por temporada

Temporada

Nortes Secas

Factor meteorológico

Temperatura máxima*

Temperatura mínima*

Oscilación térmica*

Velocidad del viento**

Nubosidad ${ }^{\circ}$

\begin{abstract}
Media
\end{abstract}
$30.40 \pm 1.25$

$19.90 \pm 3.39$

$10.5 \pm 3.04$

$2.23 \pm 1.08$

$2.68 \pm 1.94$
Variación

4.11

17.03

28.95

48.63

72.32
Media

$33.80 \pm 1.12$

$25.80 \pm 2.49$

$8.04 \pm 2.27$

$4.17 \pm 1.24$

$4.30 \pm 1.95$
Variación

3.31

9.65

28.34

29.83

45.57

Temperaturas en grados centígrados, velocidad en metros por segundo y nubosidad en escala OKT.

La variación se estimó en porcentaje con relación enl promedio de los tres días anteriores.

\section{CUADRO 7}

Efectos de las variables causales determinados por el análisis de dirección en cada temporada.

Temporada

\begin{tabular}{lcccccc}
\multicolumn{1}{c}{ Variable } & \multicolumn{3}{c}{ Nortes } & \multicolumn{3}{c}{ Secas } \\
& Directos & Indirec. & Totales & Directos & Indirec. & Totales \\
Salinidad & & & & & & \\
Temperatura del agua & .2358 & .0391 & .2749 & -.6962 & 1.0292 & .3330 \\
Profundidad & .0410 & .0673 & .1083 & .4495 & -.3622 & .0873 \\
Abundancia Batophora sp. & -.6620 & .3863 & -.2357 & 1.8352 & -2.3047 & -.4695 \\
Abundancia pastos & .4546 & -.0866 & .3680 & -.9579 & -.9899 & -1.9478 \\
Abundancia algas & -.1403 & .2008 & .0605 & -.3758 & -.6557 & -1.0315 \\
Nubosidad & .0338 & .0312 & .0650 & -1.6881 & .8724 & .8157 \\
Vientos & -.0398 & .0126 & -.0272 & .1060 & -1.0274 & -.9214 \\
Temperatura atmosférica & -.6388 & -.3221 & -.9609 & .0912 & .1652 & .2564 \\
& .3037 & .5937 & .8974 & -.3783 & .4417 & .0634
\end{tabular}


TN que en TS, el factor con menor variación en ambas temporadas fue la temperatura máxima ( $4.11 \%$ en TN y $3.31 \%$ en TS) y el de mayor variación la nubosidad $(72.32 \%$ en TN y $45.57 \%$ en TS).

Análisis de dirección: Los coeficientes de dirección del modelo propuesto así como los efectos indirectos y totales de las variables para cada temporada (Cuadro 7), determinan a la intensidad del viento y la temperatura atmosférica como las de mayor influencia en TN y al alimento disponible y al agrupamiento en TS; las de menor importancia fueron: nubosidad y alimento disponible en TN y temperatura atmosférica y del agua en TS. La validación del diagramas propuesto por el modelo de dirección reporta: $c^{2}$ significativas para el diagrama de TN (por lo que se considera que el modelo no es un buen descriptor de las relaciones causales ni del comportamiento de los datos en esta temporada) y una $c^{2}$ no significativa para el diagrama de TS (por lo que el modelo es considerado como un buen descriptor de las relaciones causales y del comportamiento de los datos en esta temporada).

\section{DISCUSION}

La discrepancia entre temporadas en el criterio de validación del diagrama propuesto por el modelo de dirección, se debe a que en TN no hay ajuste entre las covarianzas de los coeficientes de dirección y las distribuciones de los datos (Stokes 1974), lo cual es atribuible a: 1).- Las variaciones entre áreas generaron distribuciones de datos sesgadas, 2).- Los sesgos observados fueron extremos en las variables con mayores efectos totales (viento, temperatura atmosférica y $\mathrm{Ba}$ tophora sp.) y 3).- Los efectos no medidos por el análisis tiene un valor de influencia mayor que cualquiera de las variables causales consideradas (Lewis-Beck y Mohr 1976).

Con base en la discusión sobre causalidad de Biddle y Marlin (1987), se considera que la diferencia en las distribuciones de los datos entre temporadas, afecta el ajuste de las covarianzas de los coeficientes de dirección, pero no el dise- ño de causalidad propuesto por el diagrama, el cual puede ser correcto. Debido a que las distribuciones de los datos en TS ( $c^{2}$ no significativa), se apegan a los supuestos de normalidad, se puede considerar que el diagrama describe correctamente las relaciones entre variables, pero no se considera buen descriptor del comportamiento de los datos, porque cuando sus distribuciones son ses' gadas (como en TN), el diagrama causal reporta una $c^{2}$ significativa (Mitchell 1992).

Los coeficientes de dirección (efectos directos de las variables), fueron calculados a partir de las medias y desviaciones estándar de los datos de las variables, por lo que son una función de las diferencias estadísticas de los datos (Sokal y Rohlf 1981); en consecuencia, la influencia de las variables con datos que reportaron diferencias estadísticas no significativas entre áreas, fue minimizada, por lo que consideramos que se aumentaría la confiabilidad de los resultados si se realiza el cálculo de los coeficientes de dirección con la matriz de datos original sin manejo estadístico previo.

Al realizar el cálculo de los efectos indirectos de las variables, se observó que están altamente influenciados por dos factores: 1).- Su nivel de diagramación (a que variables influye y que variables la afectan) y .2).- La correlación con variables de su mismo nivel de influencia (Pedhazur 1982). Errores en cualquiera de estos factores, afecta la validación del diagrama como ocurrió en TN con la diagramación de la variable temperatura atmosférica.

En TN los resultados obtenidos no pueden ser considerados en forma cuantitativa, sin embargo, cualitativamente representan una escala jerárquica de la influencia de cada causal sobre la variable de respuesta (Asher 1983). La variable causal con mayor influencia sobre la presencia del manatí fue la intensidad del viento (conforme aumentaba la intensidad del viento, se incrementó el número de individuos en las áreas de resguardo y disminuyó en áreas con aguas abiertas) lo cual manifiesta la importancia de los nortes en la zona.

La nubosidad fue una variable que no presentó influencia significativa en la presencia del 
manatí, por lo que puede ser excluida del análisis sin que se observen variaciones significativas en el resto de los resultados. Durante TN el manatí hizo uso preferencial de zonas con profundidad menor a $2 \mathrm{~m}$, por lo que se comprueban los resultados obtenidos en la zona por Morales y Olivera $(1992,1994)$. La temperatura del agua no afectó la distribución del manatí.

Conforme el estadístico de Mitchell (1992), en TS los resultados son confiables tanto cualitativa como cuantitativamente, la mayor influencia la registró el alimento disponible, lo cual es resultado de: 1).- Mayor estabilidad en las variables meteorológicas, lo que reduce su importancia como factor limitante y 2).- Incremento en la correlación entre las variables que conforman este bloque (Batophora sp., algas y pastos). El agrupamiento presentó la segunda influencia sobre la presencia del manatí; sin embargo, examinando el procedimiento de cálculo de los coeficientes, consideramos que la influencia de esta variable al ser considerada como un bloque independiente, es resultado de procesos matemáticos y no biológicos, por lo que éste resultado debe ser considerado con reserva.

Los efectos de las factores climáticos en TS en comparación con TN disminuyeron notoriamente como consecuencia de la reducción de los sesgos en la distribución de los datos, observando que: 1).- La temperatura atmosférica presentó la menor influencia sobre la presencia del manatí pero no puede ser excluida del diagrama (como en el caso de la nubosidad), por su influencia sobre la temperatura del agua y los vientos y 2).- Los efectos directos de los vientos disminuyeron en relación con $\mathrm{TN}$, debido a una reducción en el rango de fluctuación de la intensidad y a un aumento en su velocidad promedio. Esto sugiere que la influencia del viento depende más de las variaciones drásticas que de su intensidad

La temperatura del agua en TS, al igual que en TN, reportó una baja influencia, lo cual es reflejo de la poca importancia de este factor en la distribución del manatí en la zona, al no registrarse temperaturas del agua menores a $20^{\circ} \mathrm{C}$, temperatura considerada como critica para la distribución de la especie (Shane 1981, Irvine 1983, Reynolds y Wilcox 1986) y de que al ser poco significativas las variaciones entre áreas, se consideró como una zona con temperatura del agua homogénea. Sin embargo, su importancia no debe ser subestimada, ya que dentro del diagrama causal, es una variable que afecta considerablemente los resultados de los bloques de factores abióticos, alimento disponible y agrupamiento.

Considerando los resultados obtenidos en ambas temporadas y sus limitaciones, el área de mayor uso por el manatí es la zona de la Boca de la Barra donde se une el Sistema Lagunar "Laguna Guerrero" y la Ría Cacayuc con la Bahía de Chetumal. Las variables con menor influencia en la presencia de la especie en la zona fueron: temperatura del aire y del agua (difiriendo con los resultados obtenidos en Florida E.U.A., por que en la Bahía de Chetumal la temperatura del agua fue siempre mayor a $20^{\circ} \mathrm{C}$ ) y nubosidad. Las variables con una influencia moderada fueron: salinidad (coincidiendo con los trabajos de Rathbun et al. 1982), profundidad (el manatí hizo uso preferencial de áreas con profundidades entre 0.80 y $2 \mathrm{~m}$, corroborando los resultados obtenidos en la zona por Morales y Olivera (1994) y el agrupamiento (lo cual sugiere que la zona es poco importante como área de apareamiento). Las variables con mayor influencia fueron: cambios drásticos en la intensidad del viento (con lo que se corrobora la importancia de los nortes en la zona), y el alimento disponible (por lo que se apoya la hipótesis de Colmenero y Hoz 1986; sobre la importancia del alimento en las zonas de distribución del manatí en México).

\section{AGRADECIMIENTOS}

La investigación fue financiada por el Consejo Nacional de Ciencias y Tecnología de México mediante el contrato 2017-N9301 y la Beca-Crédito 86001, y apoyada por la Fundación TELMEX mediante beca al primer autor (Folio: 3310011171). 


\section{RESUMEN}

La influencia de: temperatura atmosférica y del agua, vientos, nubosidad, profundidad, salinidad, abundancia de pastos y algas, y estructura de grupo; en la distribución espacial de Trichechus manatus manatus, fue estudiada en la Bahía de Chetumal, Quintana Roo, México; de noviembre de 1994 a junio de 1995. Las variables con menor asociación fueron: nubosidad y temperatura atmosférica y del agua (en contraste con lo reportado para la subespecie de Florida, Trichechus manatus latirostris); las variables con asociación moderada fueron: salinidad (como en Florida), profundidad (uso frecuente de profundidades entre $0.80 \mathrm{y}$ $2 \mathrm{~m}$ ) y estructura de grupo (lo cual sugiere que la zona no es importante como área de apareamiento). La distribución espacial fue asociada principalmente con cambios drásticos en la intensidad del viento y con el alimento disponible (similar a trabajos previos realizados en México).

\section{REFERENCIAS}

Altmann, J. 1974. Observational study of behavior: sampling methods. Behav. 49: 227-267.

Asher, H. B. 1983. Causal Modeling. Sage Univ. Paper series on Quant. Appli. in the Soc. Sci., series no. 07003. Sage, Los Angeles, California. 96 p.

Anonimo. 1980. El Entorno Natural. p. 9-24 In Centro de Investigación de Quintana Roo (ed.). Quintana Roo: Organización espacial. CIQRO. Puerto Morelos, Quintana Roo, México. Departamento de Geografía Económica del Instituto de Geografía de la UNAM.

Bengtson, J. L. 1983. Estimating food consumption of freeranging manatees en Florida. J. Wildl. Manage. 47: 1186-1192.

Biddle, B. J. \& M. M. Marlin. 1987. Causality, confirmation, credulity and stuctural equation modeling. Child Dev. 58: 4-17.

Bonde, R. K. 1984. Sea cows and manatees. p 295-297 In D. MacDonal (ed.). The Encyclopedia of Mammals. Facts on File, Nueva York, Nueva. York.

Campbell, H. W. \& B. Irvine. 1981. Manatee mortality during the unusually cold winter of 1976-1977, p 86-91. In R. L. Brownell\& K. Ralls (eds.). The West Indian Manatee in Florida, Proc. Workshop, Florida Dep. Nat. Resour. Orlando, Florida, 27-29 March, 1978.

Chavira-Martínez, D., J. Briseño-González, A. Negroe, J. A. Pérez-Cetina, T. Sánchez- Cabrera \& R. Hoil. 1992.
Diagnóstico de la calidad del agua en la Bahía de Chetumal, Q. Roo. Avacient. 2: 7-33.

Colmenero-Rolon, L.C. \& M. E. Hoz-Zavala. 1986 Distribución de los Manatíes, Situación y Conservación en México. Anal. Inst. Biól. UNAM. 56 (1985) Ser. Zool. (3): 955-1020.

Colmenero-Rolon, L. C., J. Azcarate C. \& E. Zárate. 1988. Estado y distribución del Manatí en Quintana Roo. Informe interno. Centro de Investigaciones de Quintana Roo - U. S. Fish and Widl. Service - Secretaría de Desarrollo Urbano y Ecología, Quintana Roo, México. $144 \mathrm{p}$.

Domming, D. P. \& L. C. Hayek. 1986. Interspecific and intraspecific morphological variation in manatees (Sirenia: Trichechus). Marine Mammal. Sci. 2 : 87-144.

Etheridge, K., G.B. Rathbun, J. A. Powell \& I. J. Kochman. 1985. Consumption of aquatic plants by the West Indian manatee. J. Aquatic Plant Manage. 23: 21-25.

Fuentes, A. I. \& A. Aguayo L. 1989. La Distribución del Manatí Trichechus manatus en el Estado de Quintana Roo, Memorias VI Simp. de Fauna Silvestre, Facultad de Medicina Veterinaria y Zootecnia -UNAM Quintana Roo, México.

Hartman, D.S. 1979. Ecology and behavior of the Manatee (Trichechus manatus) in Florida. Spec. Publi. No. 5 The Amer. Soc. Mammal. Ithaca, Nueva York. 153 p.

Husar, S. L. 1977. The West Indian manatee (Trichechus manatus). U. S. Fish and Wildl. Service, Wildl. Res. Rep. 7, Washington, D.C. 22 p.

Irvine, A. B. 1983. Manatee metabolism and its influence on distribution in Florida. Biol. Conserv. 25: 315-334.

Jefferson, T. A., S. Leatherwood \& M. A. Webber. 1993. Marine mammals of the world. FAO Species identification guide. FAO, Roma. 320 p.

Lewis-Beck, M. S. \& L. B. Mohr. 1976. Evaluating effects of independent variables. Political Method. 3: 27-47.

Lot-Helgueras, A. 1991. Vegetación y flora vascular acuática del Estado de Veracruz. Tesis de doctorado. Facultad de Ciencias, UNAM, México, D. F. 136 p.

Lluch, B. D. 1965. Further notes on the biology of the manatee. Anal. Inst. Nal. Invest. Biol. Pesquera, México 1: 405-419.

Marsh, H. \& L. W. Lefebre. 1994. Sirenian status and conservation efforts. Aquatic Mammal. 20 : 155-170. 
Mitchell, R. J. 1992. Testing Evolutionary and Ecological Hypotheses Using Path Analysis and Structural Equation Modelling. Funtional Ecol. 6: 123-129.

Morales-Vela, B. \& L. D. Olivera-Gómez. 1992. De sirenas a Manatíes. Cuadernos de divulgación No. 4 ClQRO, Quintana Roo, México. 30 p.

Morales-Vela, B. \& L. D. Olivera-Gómez. 1994. Distribución espacial y estimación poblacional de los manatíes en la Bahía de Chetumal, Quintana Roo, México. Rev. Inv. Cient. Vol.2 (No. Esp. SOMEMMA 1): 17-25.

Norris, N. J. \& Bucher, K. E. 1992. Marine algae and seagrasses from Carrie Bow Cay, Belize. Smiths. Contri. Mar. Sci. 12 : 167-223.

O' Shea, T. J., J. F. Morore \& H. I. Kochman. 1984. Contaminant concentrations in manatees in Florida. J. Wildl. Manage. 48: 741-748.

O' Shea, T., J., C. A. Beck, R. K. Bonde, H. I. Kochman \& D. K. Odell. 1985. An analysis of manatee mortality patterns in Florida, 1976-81. J. Wildl. Manage. 49: 1-11.

Pedhazur, E. J. 1982. Multiple regression in behavioral research. Holt, Rinehart and Winston, Nueva York, Nueva York. 822p.

Pielou, E. C. 1977. Mathematical Ecology. Wiley Interscience. Nueva York, Nueva York. 385p.

Powell, J. A. \& G. B. Rathbun 1984. Distribution and abundance of manatee along the northern coast of the Gulf of Mexico. Northeast Gulf Sci. 7: 1-28.

Rathbun, G. B., R.K. Bonde \& D Clay. 1982. The status of the West Indian Manatee on the Atlantic coast north of the Florida, p. 152-156. In R.R. Odum \& J. W. Guthrie (eds.). Proc. Symp. on Nongame and Endangered Wildl. Georgia Dep. Natur. Resources, Game and Fish Division, Tech. Bull. 15. 179 p.

Reynolds, J. E. 1979. Interal and external morphology of the manatee (sea cow). Anat. Rec. $193: 663$.
Reynolds, J. E. 1981. Behavior patterns in the West Indian manatee, with emphasis on feeding and diving. Florida Sci. 44 : 233-242.

Reynolds, J. E. \& J. R. Wilcox. 1986. Distribution and abundance of the West Indian manatee Trichechus manatus round selected Florida power plants following winter cold fronts: 1984-1985. Biol. Conserv. 38: 103-113.

Reynolds, J. E., D. K. Odell \& J. Reynolds. 1991. Manatee and Dugongs. Facts on File Pub. Nueva York, Nueva York. $192 \mathrm{p}$.

Rose, P. M. 1985. The West Indian Manatee, p. 540-547 In National Audubon Soc. Audubon Wildl. Rep. 1985., Nueva York, Nueva York.

Shane, S. H. 1981. Abundance, distribution and use of power plant effluents by mantee (Trichechus manatus) p. 1-24. In Florida. U. S. Fish and Widl. Service, Nat. Fish and Widl. Lab. (ed.). Brevard County. Service Prepared for Florida Power \& Light Co. Contract No. 31534-82511. NTIS Pub. No. Pb 81-147019.

Sokal, R.R. \& F. J. Rohlf. 1981. Biometry. W. H. Freeman, San francisco, California.

Stokes, D. E. 1974. Compound paths: an expository note. Amer. J. Political Sci. 18 : 191-214.

Whitehead, P. J. 1977. The former southern distribution of new world manatee (Trichechus spp.) Biol. J. Linn. Soc. 9: $165-189$

Wynne, J. M. 1985. A cheecklist to benthic marine algae of the tropical and subtropical Western Atlantic. Dep. Bio. \& Herb. Univ. of Michigan. Ann. Arbor. Michigan. p. 2239-2281

Zárate-Becerra, E. 1993. Distribución del manatí (Trichechus manatus) en la porción sur de Quintana Roo, México. Rev. Inv. Cient. UABS. Vol. 1 (No. Esp. SOMEMMA 1): 1-12. 